\title{
Analysis of Coating Structures and Interfaces in Solid Oral Dosage Forms by Three Dimensional Terahertz Pulsed Imaging
}

\author{
J. AXEL ZEITLER, ${ }^{1,2,3}$ YAOCHUN SHEN, ${ }^{1}$ COLIN BAKER, ${ }^{1}$ PHILIP F. TADAY, $^{1}$ MICHAEL PEPPER, ${ }^{1,2}$ THOMAS RADES $^{3}$ \\ ${ }^{1}$ TeraView Limited, Platinum Building, St John's Innovation Park, Cambridge, CB4 OWS, United Kingdom \\ ${ }^{2}$ Cavendish Laboratory, University of Cambridge, Madingley Road, Cambridge, CB3 OHE, United Kingdom \\ ${ }^{3}$ School of Pharmacy, University of Otago, PO Box 56, Dunedin, New Zealand
}

Received 26 May 2006; accepted 29 August 2006

Published online in Wiley InterScience (www.interscience.wiley.com). DOI 10.1002/jps.20789

\begin{abstract}
Three dimensional terahertz pulsed imaging (TPI) was evaluated as a novel tool for the nondestructive characterization of different solid oral dosage forms. The timedomain reflection signal of coherent pulsed light in the far infrared was used to investigate film-coated tablets, sugar-coated tablets, multilayered controlled release tablets, and soft gelatin capsules. It is possible to determine the spatial and statistical distribution of coating thickness in single and multiple coated products using 3D TPI. The measurements are nondestructive even for layers buried underneath other coating structures. The internal structure of coating materials can be analyzed. As the terahertz signal penetrates up to $3 \mathrm{~mm}$ into the dosage form interfaces between layers in multilayered tablets can be investigated. In soft gelatin capsules it is possible to measure the thickness of the gelatin layer and to characterize the seal between the gelatin layers for quality control. TPI is a unique approach for the nondestructive characterization and quality control of solid dosage forms. The measurements are fast and fully automated with the potential for much wider application of the technique in the process analytical technology scheme. () 2006 Wiley-Liss, Inc. and the American Pharmacists Association J Pharm Sci 96:330-340, 2007

Keywords: terahertz pulsed imaging; far infrared; structural imaging; coating analysis; film coating; sugar coating; tablets; multilayer tablet; soft gelatin capsules; process analytical technology (PAT)
\end{abstract}

\section{INTRODUCTION}

In the development of solid oral dosage forms a trend towards the development of more sophisticated coatings or layered structures to alter the release kinetics of the active pharmaceutical ingredient (API) can be observed. ${ }^{1,2}$ With the advent of more complex processes for the production of these structures and the demand for a

Correspondence to: Philip F. Taday (Telephone: +441223 435380; Fax: +44 1223 435382;

E-mail: philip.taday@teraview.com)

Journal of Pharmaceutical Sciences, Vol. 96, 330-340 (2007)

(c) 2006 Wiley-Liss, Inc. and the American Pharmacists Association rigorous process understanding by the regulatory bodies an exact characterization and control of these processes and the resulting dosage form properties becomes essential for the pharmaceutical industry. However, to date most of the methods to analyze coating properties are unspecific (e.g., weight gain during coating) or destructive to the sample (e.g., electron microscopy or infrared imaging of cross sections through a tablet). ${ }^{3-6}$

Recently, terahertz radiation has been used in pharmaceutical analysis predominantly in spectroscopy for the study of solid state properties such as polymorphism, ${ }^{7,8}$ phase transitions, ${ }^{9,10}$ 
and hydrate forms. ${ }^{11}$ The interactions of terahertz radiation with organic molecular crystals have been studied and first interpretations of the spectral features have been made. ${ }^{12,13}$ It was observed that crystalline materials generally exhibit strong absorption features in this frequency range whereas predominantly amorphous materials, such as many pharmaceutical excipients are semitransparent to terahertz radiation. This enables a reasonable penetration of the terahertz pulse into the sample specimen which in turn allows the three dimensional spatial resolution of interfaces within the sample. A first review on the field was published recently. ${ }^{14}$

In an earlier work we have demonstrated how terahertz pulsed imaging (TPI), using pulsed coherent light in the far infrared region of the electromagnetic spectrum, can be used for the nondestructive analysis of coated tablets. ${ }^{15}$ In that study the sample was kept stationary and pulses of terahertz radiation were focused onto the tablet. The surface was scanned with the terahertz beam. Using this approach the sample area is quite restricted due to size and curvature of a typical biconvex pharmaceutical tablet. Additionally the sample would have to be turned manually in order to examine both faces of a tablet. To overcome this limitation we have developed a robotic system for the fully automated scan of a typical pharmaceutical solid dosage form.

In the present study, this new setup was evaluated for its use as a novel tool for the nondestructive characterization of different solid oral dosage forms.

\section{EXPERIMENTAL}

\section{Materials}

Tablet A: Dicolfenac-ratiopharm ${ }^{\circledR} 50$ (Ratiopharm GmbH, Germany) enteric-coated tablets containing $50 \mathrm{mg}$ diclofenac, coating material based on 1:1 copolymer of 2-methacrylic acid and ethyl acrylate (batch \#1 12 tablets, batch \#2 30 tablets). Tablet B: Sinupret ${ }^{\circledR}$ forte Dragees (Bionorica, Germany) sugar-coated tablets containing a mixture of different herbal drugs. Tablet C: Diclofenac-ratiopharm ${ }^{\circledR}$ uno (Ratiopharm GmbH, Germany) multilayered controlled release tablets based on the Geomatrix ${ }^{\circledR}$ quick slow release technology. ${ }^{16}$ Capsule A: Spalt liqua (Whitehall-Much GmbH, Germany) softcaps containing $200 \mathrm{mg}$ ibuprofen in a liquid formulation.
All sample tablets originated from the same production batch if not stated otherwise.

\section{Three Dimensional Terahertz Pulsed Imaging}

In this study, the solid dosage forms were analyzed using a TPI imaga2000 system (TeraView, Cambridge, UK). The generation of terahertz radiation in this system is based on a photoconductive switch setup as described previously. ${ }^{15}$ It is adapted to meet the specific needs of this application. Terahertz photoconductive emitters rely on the production of few-cycle terahertz pulses using an ultrafast (femtosecond) laser to excite a biased photoconductive antenna. This technique is inherently broadband, with the emitted power distributed over several $\mathrm{THz}$ (typically $60 \mathrm{GHz}-3 \mathrm{THz}$, corresponding to 2$100 \mathrm{~cm}^{-1}$ ). Pulsed terahertz emission in photoconductive antennas is produced when the current density, $j$, of a biased semiconductor is modulated on subpicosecond timescales $E_{\mathrm{THz}} \propto \mathrm{d} j /$ $\mathrm{d} t .{ }^{17}$ The change in current density mainly arises from the acceleration of photogenerated carriers under an external electric field. Coherent detection of the incident $\mathrm{THz}$ radiation can be performed in a similar photoconductive antenna circuit. ${ }^{18}$ By gating the photoconductive gap with a femtosecond pulse synchronized to the terahertz pulse, a dc signal that is proportional to the terahertz electric field may be measured. Hence, both the amplitude and phase of the incident $\mathrm{THz}$ wave can be obtained. Further, by varying the optical path length to the receiver, the entire terahertz time-domain can be sampled. The ability to measure the electric field of the signal in this setup is in contrast to the signal detection employed in most other spectroscopic techniques where only the signal intensity is recorded. The resulting terahertz waveform therefore exhibits positive as well as negative signal.

In this particular system (see Fig. 1), the beam from the ultrafast femtosecond laser is split into two components, a pump beam and probe beam, with one used to illuminate the emitter and receiver respectively. A rapid scanning delay line, is then incorporated into the probe beam to vary the difference in optical delay around zero between the incoming terahertz pulse and the probe laser pulse at the detector, thus allowing both the delay position and the lock-in output of the modulated external electrical field to be digitized and reinterpolated to obtain the terahertz field as a function of optical delay in real time. The two laser beams are 


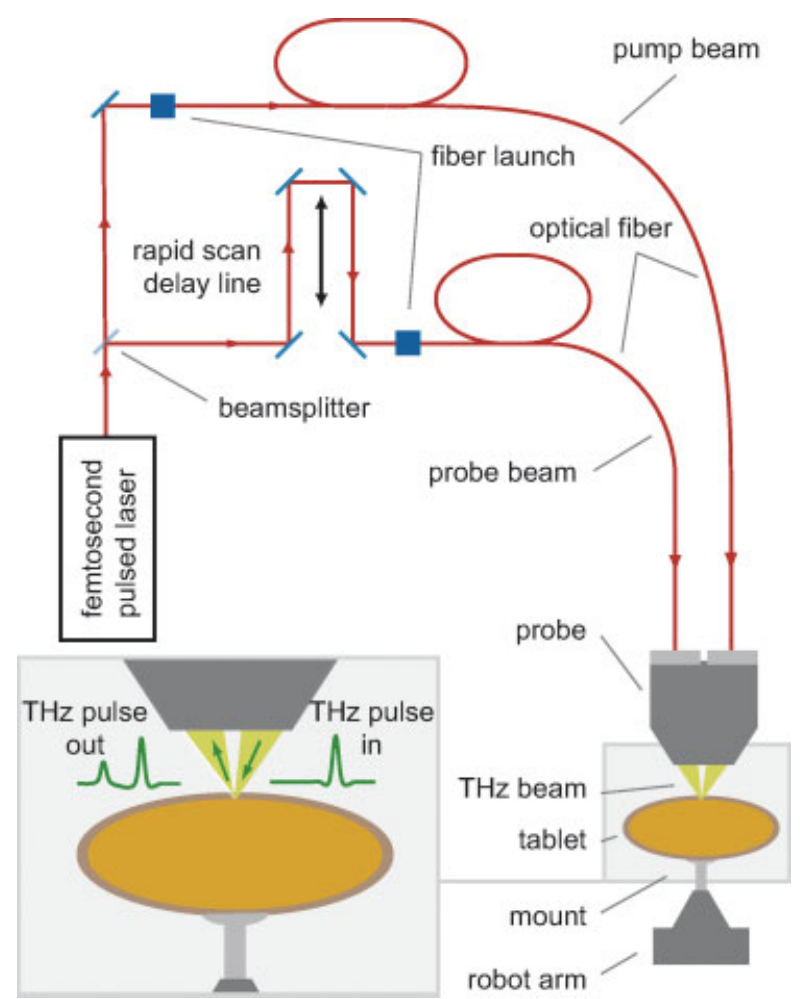

Figure 1. Schematic of the TPI imaga2000 system. The insert shows a schematic diagram of the experimental arrangement to examine coatings, in this case for two interfaces. A reflected terahertz signal occurs whenever there is a change in boundary conditions. [Color figure can be seen in the online version of this article, available on the website, www.interscience. wiley.com.]

launched into separate optical fibers to link the TPI core unit to the separate solid dosage form scanning unit. After leaving the output from the optical fibers in the solid dosage form scanning unit, the laser beam is focused onto the gaps of the photoconductive emitter and detector using an optical lens. A modulated bias was applied across the emitter electrodes, and the gated output signal from the receiver was fed to a lock-in amplifier, referenced to a modulation in the emitter bias. A signal strength of $>60 \mathrm{~dB}$ in the frequency domain was demonstrated using time-gated and lock-in detection. $^{19}$

The generated terahertz output from the emitter is coupled into free-space using a highresistivity silicon lens, which, in combination with silicon probe optic, allows the terahertz beam to be focused to a diffraction limited spot. The focus of the terahertz spot is incident on the solid dosage form under test, $5 \mathrm{~mm}$ in front of the silicon optic. The reflected light from the solid dosage form is collected by the same silicon optic, and coupled from free-space into the receiver, using a second silicon lens in contact with the rear of the receiver chip. All terahertz generation and detection components together with the optics are assembled into a probe unit (Fig. 1).

Scanning the solid dosage form over a laser gauge by a six-axis robot arm produces a surface map of the solid dosage form under test. This profile of the solid dosage form surface is then used to scan the solid dosage form across the terahertz beam until the entire surface is covered, producing a 3D terahertz image of the solid dosage form. The terahertz pulses incident on a solid dosage form, give rise to echoes or reflections from different buried layers (for instance at the coating-API interface).

Figure 1 shows a schematic diagram of the TPI imaga2000 measurement. The terahertz pulse is reflected first off the front surface and then from any subsurface structure within the solid dosage form resulting in multiple pulses returning to the detector.

\section{Data Analysis}

Reflections or echoes arise whenever there is either a chemical or structural change in the solid dosage form leading to a change in the refractive index and/or absorption coefficient of the material in the terahertz. The signal to noise ratio associated with the technique allows small changes in refractive index to be detected and imaged.

Impulse functions are obtained from the raw terahertz reflected waveform by deconvolving the system response. The deconvolution is performed by dividing, in the frequency domain, the raw terahertz waveform reflected from the solid dosage form by a reference waveform recorded from a mirror surface. A numerical bandpass filter is applied to remove high- and low-frequency noise. Using this simple deconvolution process the terahertz pulse duration of $<200 \mathrm{fs}$ produces an axial resolution of $38 \mu \mathrm{m}$ depending on the refractive index of the material under investigation (Fig. 2). For samples below this resolution limit the measured values increase as the interface search algorithm used for the analysis of the data cannot locate a resolved peak in the terahertz waveform. Any pixels that would fall into this range are excluded from the terahertz image analysis.

With knowledge of the refractive index of the coating material values of average coating thickness as well as histograms and maps of coating 


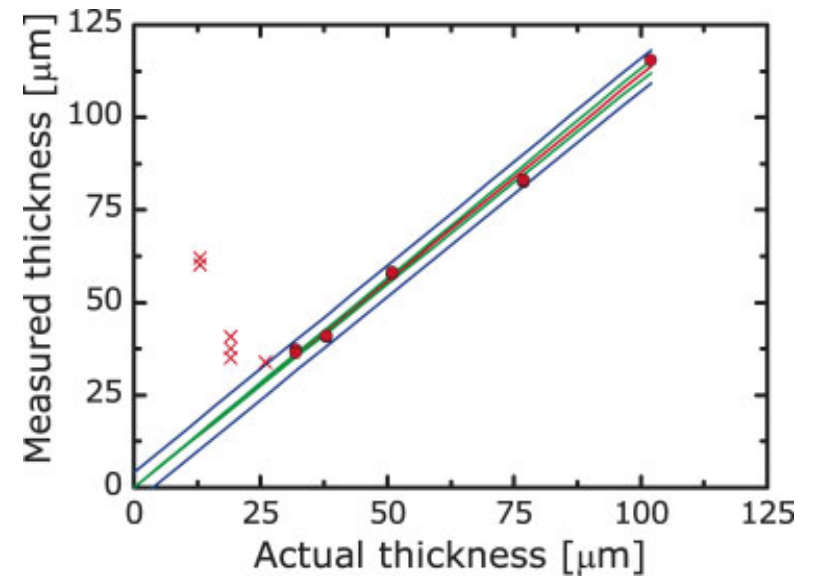

Figure 2. Thickness calibration. Plot of the measured thickness with the TPI imaga2000 against the actual thickness of different polyethylene sheets $(13-102 \mu \mathrm{m})$. Data point labeled $\bigcirc$ correspond to measurements above the resolution limit, below the resolution limit. [Color figure can be seen in the online version of this article, available on the website, www.interscience. wiley.com.]

uniformity can been produced from TPI analysis. ${ }^{15}$ In this work, the refractive index of the coatings is assumed to be 1.53-as determined from measurements of typical coating materials taken on a TPS spectra1000 (TeraView) transmission spectrometer.

\section{RESULTS AND DISCUSSION}

\section{Film-Coated Tablets}

Twelve different tablets of the same production batch of tablet A (Fig. 3A,B) were investigated. The initial full scan of both sides and the center band of a single tablet takes about 20-50 min and is completely automated. Once a surface model of the tablet has been built during the initial scan, the subsequent scans for both sides of a sample of Tablet A take approximately $20 \mathrm{~min}$ each.

The time-domain waveform of the terahertz signal (Fig. 4A) is reflecting information about the structure of the tablet. In the terahertz waveform the initial Gaussian peak is due to the partial reflection of the terahertz pulse from the surface of the tablet. Part of the terahertz radiation propagates further into the tablet as most pharmaceutical excipients are semitransparent in this spectral range. The subsequent minimum in the waveform is due to the reflection of the remaining terahertz pulse from the buried interface between the outer coating layer (cosmetic coating) and the

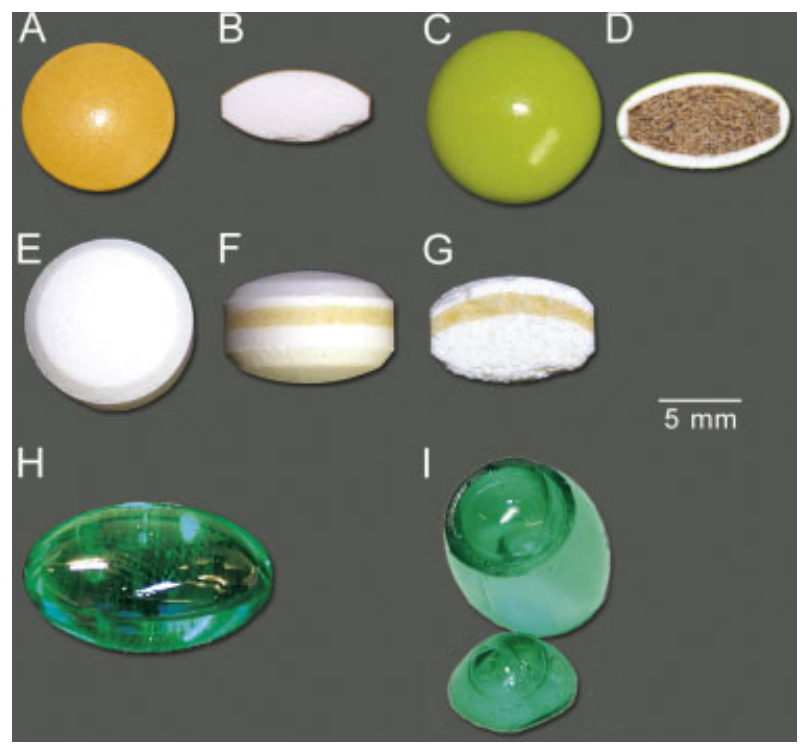

Figure 3. Photograph of the different solid oral dosage forms studied by TPI. (A) Top view of Tablet A, an enteric-coated tablet; (B) cross section through Tablet A; (C) top view of Tablet B, a sugar-coated tablet; (D) cross section through Tablet B; (E) top view of Tablet $\mathrm{C}$, a trilayered controlled release tablet; $(\mathrm{F})$ side view of Tablet C; (G) cross section through Tablet C; (H) Capsule A, a soft gelatin capsule; and (I) cross section through Capsule A. [Color figure can be seen in the online version of this article, available on the website, www. interscience.wiley.com.]

inner coating layer (enteric coating). The signal is negative indicating that the refractive index of the inner coating layer is smaller than that of the outer (pigmented) coating layer $\left(n_{2}<n_{1}\right)$. The second negative minimum in the waveform is corresponding to the reflection originating from the interface between the inner coating layer and the tablet core. The tablet core has a lower refractive index than the inner coating layer $\left(n_{3}<n_{2}\right)$ hence again a negative peak can be observed.

From the time delay between the peak of the surface reflection and the peak of the reflection from the coat/core interface the thickness of the layers can be calculated by a simple time-of-flight calculation. Using this approach the thickness of each coating layer is calculated for each pixel on the tablet surface. This information can then be analyzed in terms of the distribution of the layer thickness as a histogram and as a false color image illustrating the spatial distribution over the tablet surface (Fig. 5).

In Figure 5 the terahertz images and coating thickness distributions for one side of three tablets of this set are highlighted. All three tablets (A5, 


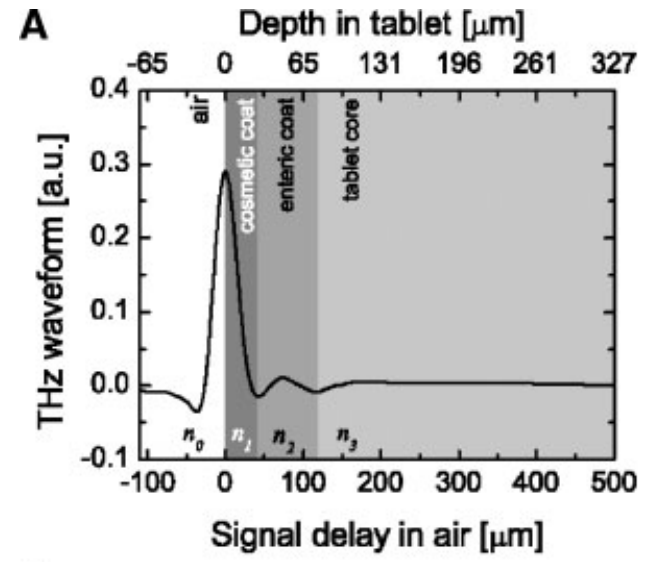

B
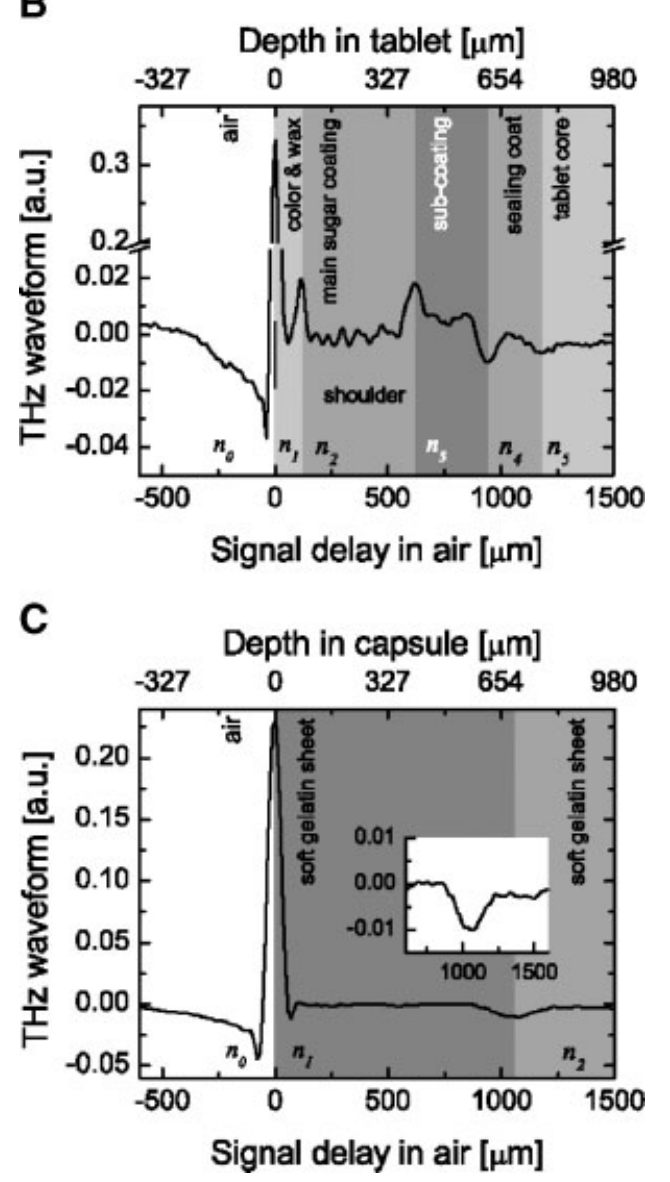

Figure 4. Terahertz waveform of (A) Tablet A1, a film-coated tablet. $n_{1}$ refractive index of the outer coating material, $n_{2}$ refractive index of the inner coating material, and $n_{3}$ refractive index of the tablet core; (B) Tablet B; (C) a pulse reflected from the body of Capsule A.

A8, and A12) exhibit a uniform coating thickness distribution for both coating layers. The minimum average layer thickness for the outer coating layer is $38 \mu \mathrm{m}$ (Fig. 5A) and the maximum layer thickness is $49 \mu \mathrm{m}$ (Fig. 5I). For the inner coating layer the average layer thickness ranges from 55 to $76 \mu \mathrm{m}$. No holes in either of the coating layers were detected.

To investigate the reproducibility of the coating quality 30 tablets of a second production batch of Tablet A (batch \#2) were analyzed. Again, no holes in either of the coating structures were observed. Table 1 and Figure 6 show the results from the coating analysis of batch \#1 and \#2. A two sample independent $t$-test was performed and no significant difference in coating thickness was found between the two batches at significance level $\alpha=0.05$ ( $p=0.12738$ for the outer layer and $p=0.54883$ for the inner layer).

In contrast to other methods for the analysis of coating thickness such as NIR spectroscopy ${ }^{20}$ TPI provides a direct measurement of the coating thickness rather than an estimate of the thickness based on a chemometric model. No model calibration and maintenance is required for the terahertz measurements. The advantage of the NIR spectroscopic method lies in its applicability for real time at-line coating process monitoring. ${ }^{21}$ Using this approach it is possible to estimate the average coating thickness of a bulk of moving tablets based on the reflection signal. TPI however, is able to provide much more detailed information on the spatial distribution of the coating over the whole surface of the tablet. Holes in enteric coats can be detected even though they might be covered with a cosmetic coating layer. With an acquisition time for a single point measurement of $50 \mathrm{~ms}$, and the potential to substantially reduce this time, TPI could also be used for the online automatic inspection of every coated tablet before blistering or even for inline process control during the coating process.

\section{Sugar-Coated Tablets}

Using the example of Tablet B (Fig. 3C,D) the structural information in terahertz images of sugar-coated tablets was evaluated. Compared to the film coating process the sugar coating process is much more elaborate. A number of different steps such as the application of a sealing coat layer, one to several sub-coatings, smoothing steps, color coating, finishing, and polishing are necessary during sugar coating. The whole process is discontinuous and even though it is performed by highly skilled and experienced personnel the manual nature of the process may lead to a lot of batch to batch variability. In 
A

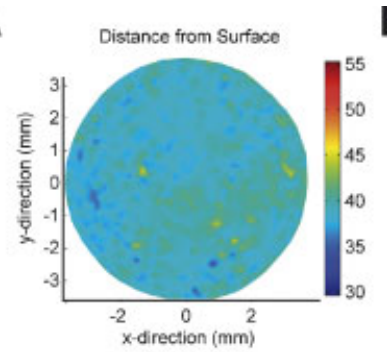

B

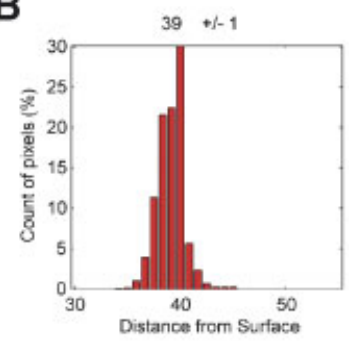

$\mathbf{E}$

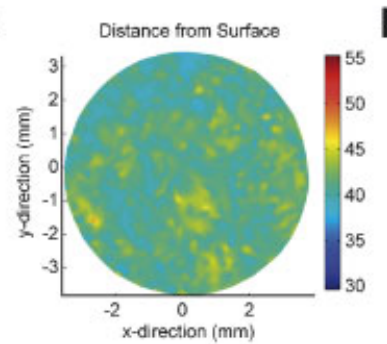

I

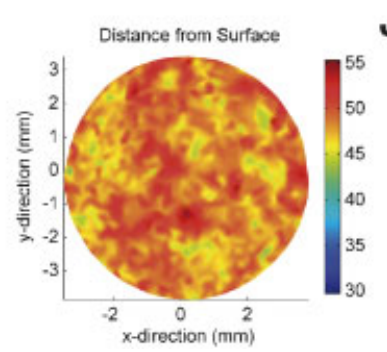

$\mathbf{F}$

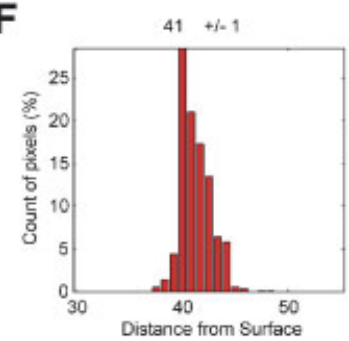

$\mathbf{J}$

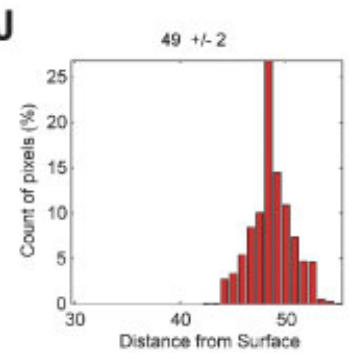

C
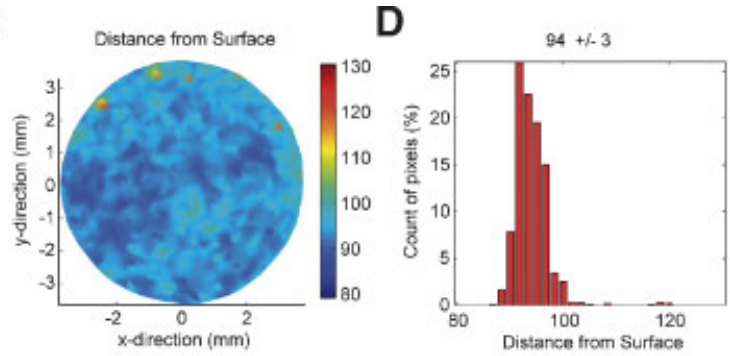

G
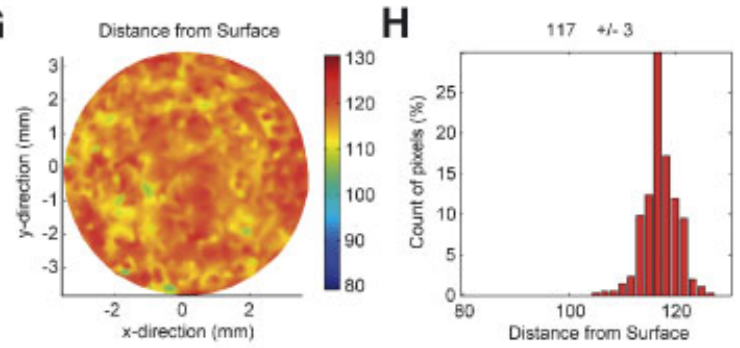

$\mathbf{K}$

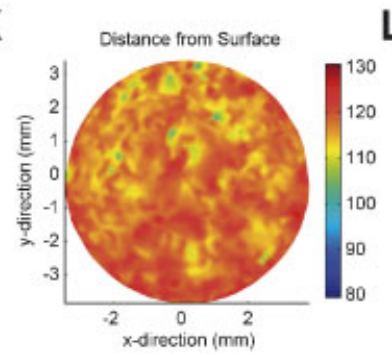

$\mathbf{L}$

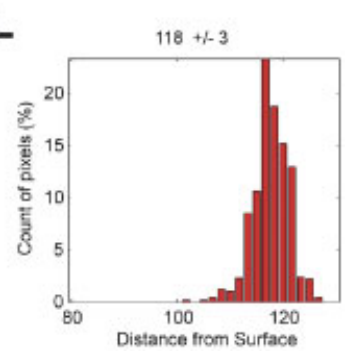

Figure 5. False color images and histograms of the coating thickness distribution for both coating layers of Tablet A (scale in $\mu \mathrm{m}$ ); (A) spatial distribution of cosmetic coating layer Tablet A5a; (B) coating thickness distribution for the cosmetic coating in Tablet A5a; (C) spatial distribution of enteric coating layer Tablet A5a; (D) coating thickness distribution for the enteric coating in Tablet A5a; (E-H) Tablet A8b; (I-L) Tablet A12b. [Color figure can be seen in the online version of this article, available on the website, www.interscience.wiley.com.]

contrast to the terahertz waveform of a filmcoated tablet (Fig. 4A) the signal from a sugarcoated product is much more complex (Fig. 4B). A number of different features can be discerned that correspond very well to the properties of a sugar-coated tablet. The materials used for the sugar coating process have different refractive indices. In addition, between most of the steps of the process the coating material on the surface of the tablet is allowed to partly or fully dry.

Table 1. Results From the Statistical Analysis of the Coating Thickness Analysis of Batch \#1 and \#2 for Tablet A

\begin{tabular}{lcccc}
\hline & $\begin{array}{c}\text { Batch \#1 } \\
\text { Outer Layer }\end{array}$ & $\begin{array}{c}\text { Batch \#1 } \\
\text { Inner Layer }\end{array}$ & $\begin{array}{c}\text { Batch \#2 } \\
\text { Outer Layer }\end{array}$ & $\begin{array}{c}\text { Batch \#2 } \\
\text { Inner Layer }\end{array}$ \\
\hline$n$ & 24 & 24 & 60 & 60 \\
Mean thickness $(\mu \mathrm{m})$ & 42 & 67 & 43 & 66 \\
SD $(\mu \mathrm{m})$ & 3 & 7 & 2 & 4 \\
Standard error of the mean $(\mu \mathrm{m})$ & 0.6 & 1.4 & 0.3 & 0.5 \\
P25 $(\mu \mathrm{m})$ & 40 & 59 & 41 & 63 \\
P75 $(\mu \mathrm{m})$ & 43 & 72 & 44 & 68 \\
Minimum thickness $(\mu \mathrm{m})$ & 38 & 53 & 39 & 55 \\
Maximum thickness $(\mu \mathrm{m})$ & 49 & 76 & 49 & 75 \\
Median $(\mu \mathrm{m})$ & 41.5 & 69.5 & 43 & 66 \\
\hline
\end{tabular}


A

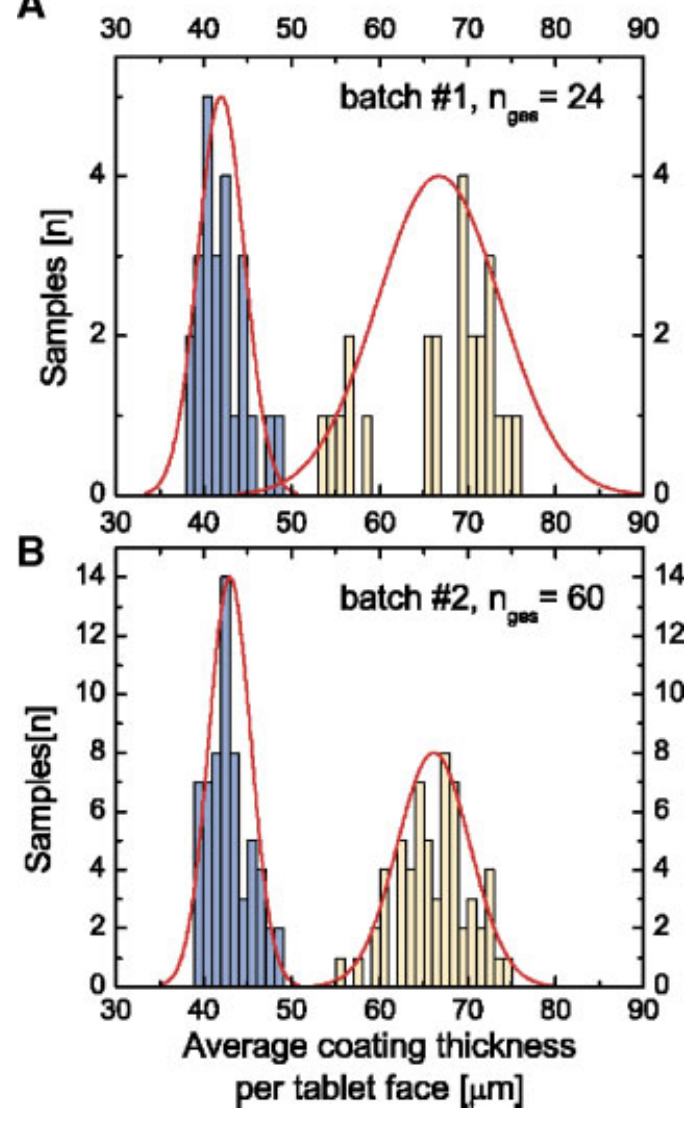

Figure 6. Histogram of the average coating thickness for both layers of batch \#1 (A) and batch \#2 (B) of the filmcoated tablets. [Color figure can be seen in the online version of this article, available on the website, www. interscience.wiley.com.]

Consequently a number of different interfaces with varying signal height can be observed in the time-domain waveform (Fig. 4B).

The color coating layer generates a very distinct positive terahertz signal between 45 and $90 \mu \mathrm{m}$ with its peak at around $70 \mu \mathrm{m}$. A shoulder on this peak at around $52 \mu \mathrm{m}$ could be due to the wax layer from the polishing process. At longer delay times the signal is followed by the terahertz signature of the main sugar coating layer $(90-360 \mu \mathrm{m})$. This layer, which represents the bulk of the sugar coat, exhibits a fine structure that is most likely caused by the discontinuous nature of the coating process. Very subtle changes in refractive index are caused by interfaces due to drying the tablets after each application of sugar suspension during this "smoothing" step. Here, at least eight times the tablets were allowed to dry during the application of the main sugar coat. Three more very distinct interfaces can be identified in the terahertz signal: a positive peak at about $410 \mu \mathrm{m}$ below the tablet surface, a negative peak at about $620 \mu \mathrm{m}$, and another negative peak at about $780 \mu \mathrm{m}$. These peaks mark the interfaces of the sub-coat and the sealing layer that are applied to the tablet core. The refractive index of the sub-coating layer $\left(n_{3}\right)$ is higher than the refractive index of the main sugar coat $\left(n_{2}\right)$, leading to a positive peak at the interface. The high refractive index of the sub-coating layer can be explained with the high content of material such as talk, kaolin, and calcium carbonate that is usually employed in this step to provide a good fundament for the main sugar coat and to cover any sharp corners or edges of the tablet core. Furthermore $n_{3}$ is also higher than the refractive index of the sealing coat layer $\left(n_{4}\right)$, which acts as a moisture barrier for the initial tablet core during the subsequent coating process, leading to a negative peak at this interface. The fact that the peak of the interface between the sealing coat layer and the tablet core at about $780 \mu \mathrm{m}$ is negative corresponds well with our previous observations that the tablet core material usually has a lower refractive index than the highly polymeric sealing coat material. In general the signal intensity decreases and the peaks broaden the deeper the interfaces are located below the surface. However, even though this tablet exhibits a very complex coating structure the terahertz signal is strong enough to resolve all coating layers and penetrate to the tablet core. At longer delay the signal from the tablet core is not as smooth as from the coating structure with noise in the signal due to scattering effects originating from the porosity of the tablet core.

Using the data acquired by the terahertz scan over the tablet surface a three dimensional depth profile of the spatial distribution of the different interfaces can be generated (Fig. 7). In this figure the curved tablet surface is projected into a plane to facilitate the analysis. The buried interfaces from the different coating layers are all depicted by their distance from the tablet surface. Image contrast is generated directly from the strength of the terahertz electric field. The cross sections reveal that the sugar coat at the rim of the tablet core is thinner compared to the thickness of the coat in the center of the tablet. This is due to the different shape of the initial tablet core and the finished sugar-coated tablet as the tablet cores are produced on a standard tablet press using a tooling with deep concave geometry which facilitate the build up of coating material during the sugar coating process. The tablet core geometry can be verified in the optical image of a cross section through the tablet (Fig. 3D). 

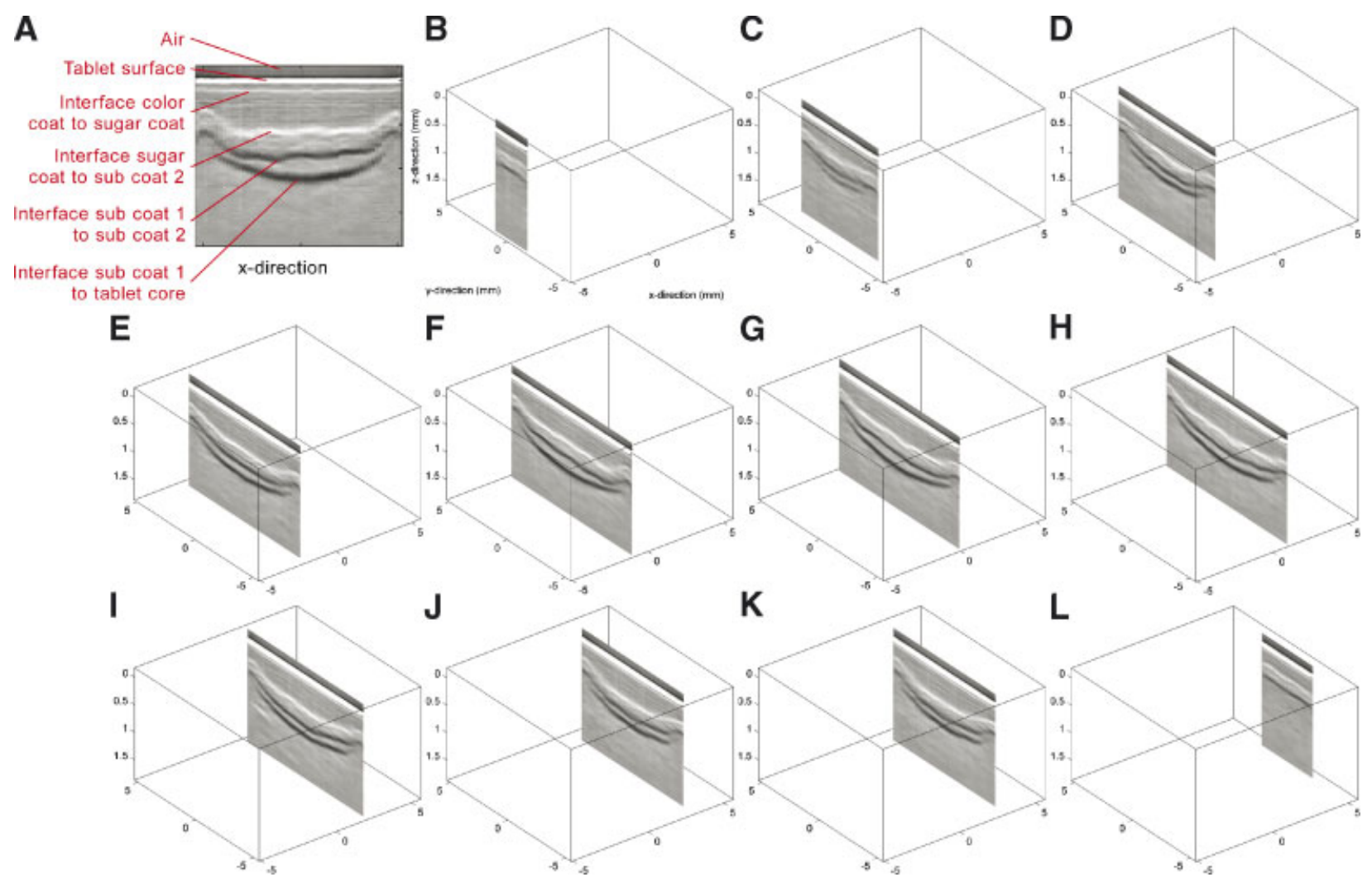

Figure 7. Terahertz images of the cross sections through Tablet B in x-direction. The tablet surface is projected into a flat plane. [Color figure can be seen in the online version of this article, available on the website, www.interscience.wiley.com.]

Even though the outer surface of the tablet and the tablet core both have a very smooth and even surface this is not the case for the layers in between. The terahertz images reveal that the first two layers (called the sealing coat and subcoating layer here) exhibit quite uneven surfaces. Only after the application of the sugar coating layer the surface appears even again.

\section{Controlled Release Tablets}

Tablet $\mathrm{C}$ was chosen as an example for the analysis of a controlled release dosage form by TPI. This tablet consists of three layers in total with API in the middle layer (yellow) and one white layer on top and bottom respectively for the control of the API release kinetics (Fig. 3E-G). Here, terahertz imaging can be used to characterize the interface between the different tablet layers.

In Figure 8 the interface between the top white layer and the middle yellow layer is characterized. This first internal interface is detected at about $700 \mu \mathrm{m}$ below the top surface of the tablet. At this interface the reflection strength of the terahertz signal is strong and a terahertz reflection can be observed for all pixels of the image. The images show that the middle layer is not flat, as a visual inspection of the tablet would suggest, but follows the curvature of the outside surface. This correlates very well with the observation of the cross section through the tablet (Fig. 3G).

The terahertz signal is strong enough to penetrate through the first two layers to the third interface between the yellow middle layer and the bottom white layer and reflect back to the detector. Even though this back reflection is very weak it can be detected with sufficient signal-to-noise. The interface between these two layers is located about $2000 \mu \mathrm{m}$ below the tablet surface. A three dimensional model of all interfaces in the tablet as detected from the terahertz beam through the top surface can be generated (Fig. 9 and video sequence in the supplementary materials).

\section{Soft Gelatin Capsules}

Figure 4C shows the terahertz time-domain waveform of a typical reflection signal from a soft gelatin capsule. The first peak that can be 

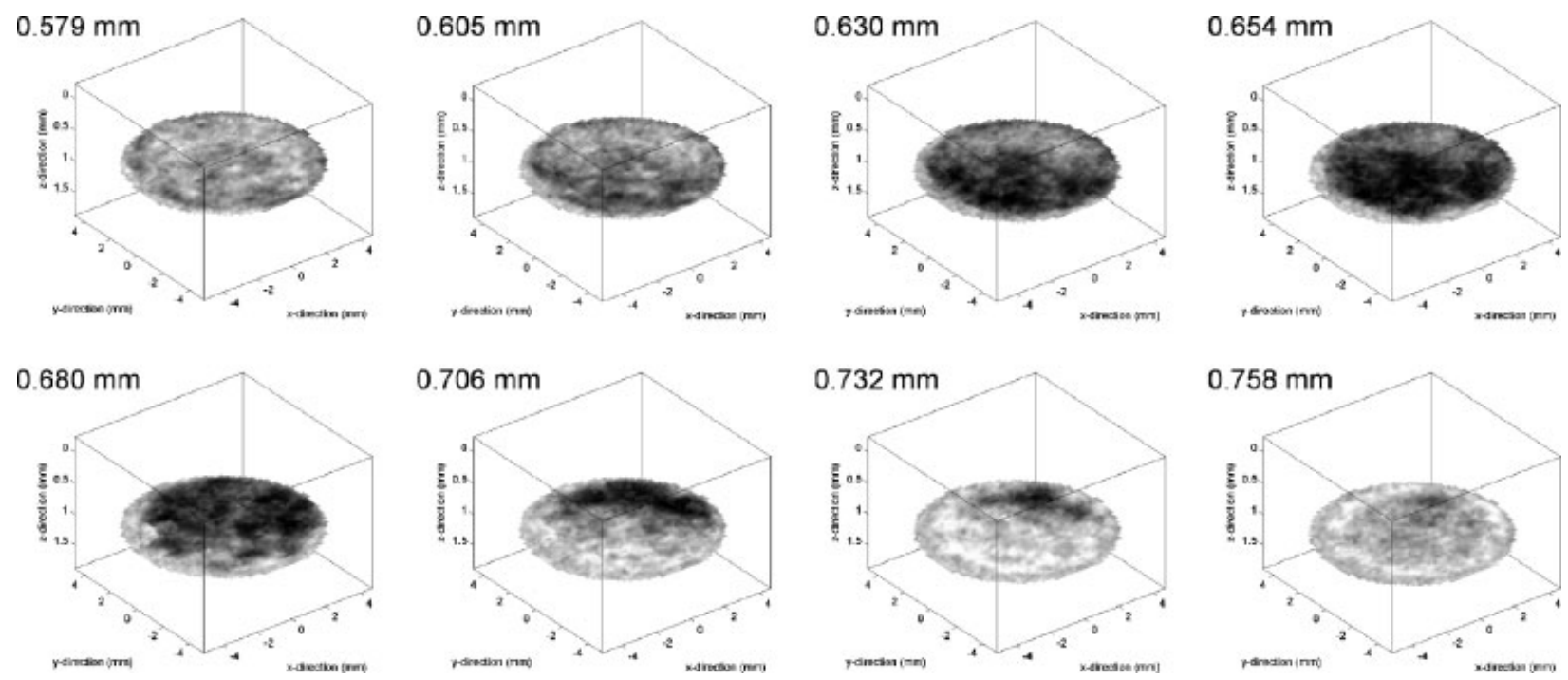

Figure 8. Terahertz images of the cross section through Tablet $\mathrm{C}$ in $\mathrm{z}$-direction. The interface form the first layer to the middle layer is highlighted.

observed is due to the back reflection of the terahertz pulse from the outer surface of the gelatin layer. Part of the pulse however propagates into the sample and a second back reflection from the interface between the gelatin layer and the liquid filling can be registered from a depth of about $700 \mu \mathrm{m}$. This reflection signal is negative indicating the transition of the terahertz light into a less dense optical medium. Compared to the signal from the air-gelatin interface the peak at the gelatin-liquid interface is much broader and less intense. The time delay between the two peaks can be used to calculate the thickness of the gelatin sheet for every single recorded pixel (Fig. 10A). The terahertz images reveal that the thicknesses of the two soft gelatin sheets that were used to manufacture the capsule are different. This finding can be confirmed by cutting the capsule open after the nondestructive TPI analysis (Fig. 3I). One half has an average thickness of about $520 \mu \mathrm{m}$ whereas the other half has an average thickness of about $690 \mu \mathrm{m}$ (Fig. 10A).

By illustrating the relative peak strength of the reflection signals in the terahertz waveform (interface index, II) rather than the time delay, the area of the seam between the two gelatin sheets can be clearly distinguished from the rest of the capsule (Fig. 10B). At the seam the peak signal strength ratio is in average about 1.2 whereas for the rest of the capsule a ratio with an average about 2.5 can be measured regardless of the gelatin sheet thickness. The layer thickness analysis of this area reveals that the gelatin structure at the seam between the two sheets is much thinner than the two gelatin sheets themselves. The thickness of
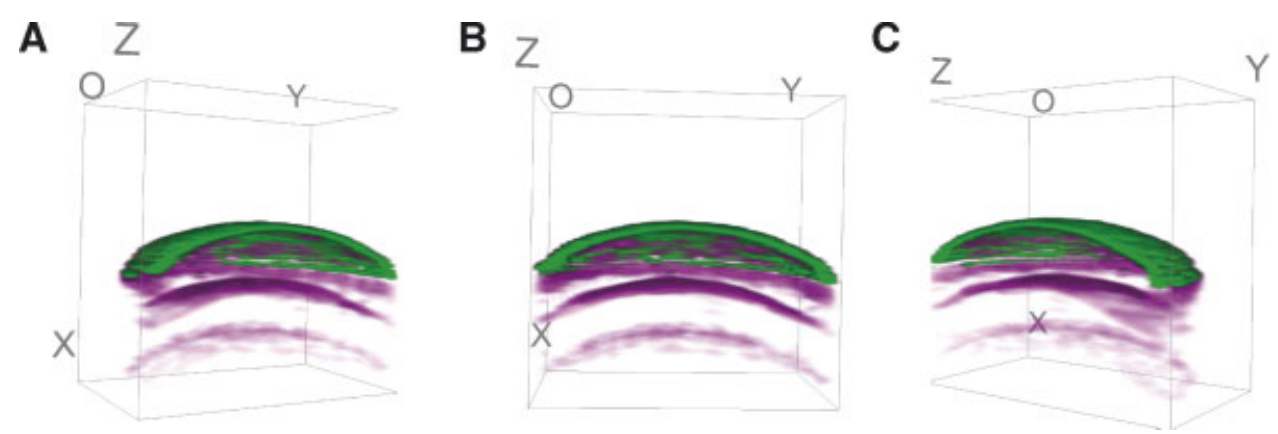

Figure 9. Three dimensional model of the interfaces in Tablet $\mathrm{C}$ as detected from the top surface (green). The purple structures mark the interfaces between the different layers of the tablet. [Color figure can be seen in the online version of this article, available on the website, www.interscience.wiley.com.] 
A

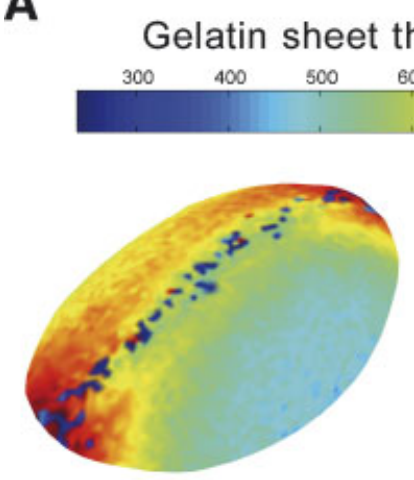

hickness $(\mu \mathrm{m})$
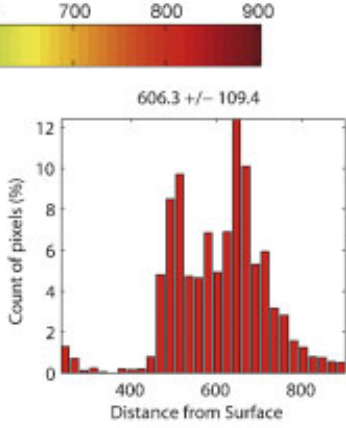

B

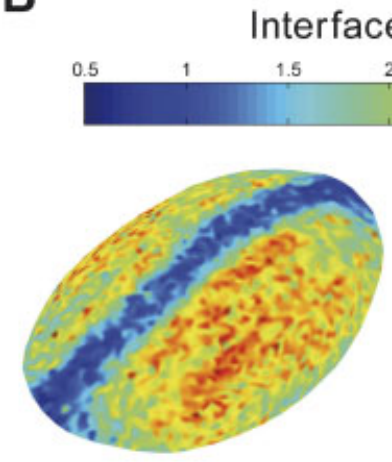

\section{Interface index}

$2 \quad 2.5$
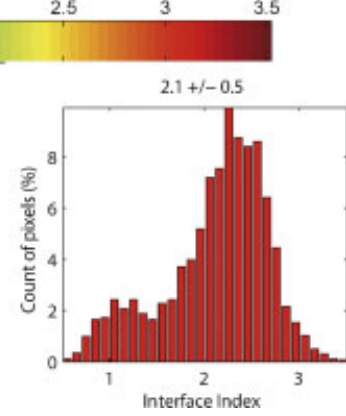

Figure 10. Spatial distribution and histogram of the gelatine layer thickness (A) and interface index (B) over the surface of the capsule. [Color figure can be seen in the online version of this article, available on the website, www.interscience.wiley.com.]

the soft gelatin in this area of the capsule is only about $250 \mu \mathrm{m}$.

Further data processing allows to subtract the gelatin layer from the $3 \mathrm{D}$ images and to focus on the liquid filling of the capsule (Fig. 11). Here again, the area of the seam can be clearly distinguished from the body of the capsule as the liquid can penetrate further into areas of the seam compared to the rest of the capsule. At present the spatial resolution of the images is in the order of a few 100 microns. Therefore, the liquid filling at

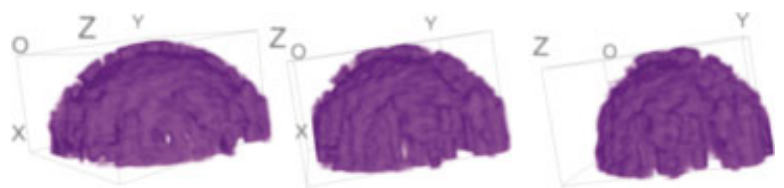

Figure 11. Model of the liquid filling in the soft gelatine capsule after subtraction of the gelatine layer based on the TPI signal. [Color figure can be seen in the online version of this article, available on the website, www.interscience.wiley.com.] the area of the seam could well be shaped like a continuous rim rather than as spiked as depicted.

\section{CONCLUSION}

In this study, a new concept for the three dimensional structural analysis of solid dosage forms has been described and evaluated. We were able to present different examples how terahertz radiation can be used for the nondestructive investigation of coating structures, interfaces between different layers in multilayered tablets, and critical quality parameters in soft gelatin capsules. TPI is a unique novel tool for the development of advanced dosage forms, quality control in existing products, and the potential for at line inspection in a production environment. Further studies will have to evaluate the influences of various coating formulations and other variables, such as lakes, imprinting, and water content.

\section{REFERENCES}

1. Kottke MJ, Rudnic EM. 2002. Tablet dosage forms. In: Banker GS, Rhodes CT, editors. Modern pharmaceutics, 4th ed. New York: Marcel Dekker, 287-334p.

2. Jantzen GM, Robinson JR. 2002. Sustained and controlled-release drug delivery systems. In: Banker GS, Rhodes CT, editors. Modern pharmaceutics, ed. New York: Marcel Dekker, 501-528p.

3. Mouget $\mathrm{Y}$, Gosselin P, Tourigny M, Bechard S. 2003. Three-dimensional analyses of tablet content and film coating uniformity by laser-induced breakdown spectroscopy (LIBS). Am Lab News 35:20-22.

4. Ruotsalainen M, Heinamaki J, Guo HX, Laitinen N, Yliruusi J. 2003. A novel technique for imaging film coating defects in the film-core interface and surface of coated tablets. Eur J Pharm Biopharm 56:381-388.

5. Reich G. 2005. Near-infrared spectroscopy and imaging: Basic principles and pharmaceutical applications. Adv Drug Deliv Rev 57:1109-1143.

6. Sinka IC, Burch SF, Tweed JH, Cunningham JC. 2004. Measurement of density variations in tablets using X-ray computed tomography. Int J Pharm 271:215-224.

7. Strachan CJ, Rades T, Newnham DA, Gordon KC, Pepper M, Taday PF. 2004. Using terahertz pulsed spectroscopy to study crystallinity of pharmaceutical materials. Chem Phys Lett 390:20-24.

8. Strachan CJ, Taday PF, Newnham DA, Gordon KC, Zeitler JA, Pepper M, Rades T. 2005. Using terahertz pulsed spectroscopy to quantify 
pharmaceutical polymorphism and crystallinity. J Pharm Sci 94:837-846.

9. Zeitler JA, Newnham DA, Taday PF, Strachan CJ, Pepper M, Gordon KC, Rades T. 2005. Temperature dependent terahertz pulsed spectroscopy of carbamazepine. Thermochim Acta 436:71-77.

10. Zeitler JA, Newnham DA, Taday PF, Threlfall TL, Lancaster RW, Berg RW, Strachan CJ, Pepper M, Gordon KC, Rades T. 2006. Characterization of temperature induced phase transitions in the five polymorphic forms of sulfathiazole by terahertz pulsed spectroscopy and differential scanning calorimetry. J Pharm Sci 95:2486-2498.

11. Zeitler JA, Kogermann K, Rantanen J, Rades T, Taday PF, Pepper M, Aaltonen J, Strachan CJ. 2006. Drug hydrate systems and dehydration processes studied by terahertz pulsed spectroscopy. Int J Pharm submitted.

12. Day GM, Zeitler JA, Jones W, Rades T, Taday PF. 2006. Understanding the influence of polymorphism on phonon spectra: Lattice dynamics calculations and terahertz spectroscopy of carbamazepine. J Phys Chem B 110:447-456.

13. Allis DG, Prokhorova DA, Korter TM. 2006. Solidstate modeling of the terahertz spectrum of the high explosive HMX. J Phys Chem A 110:19511959 .

14. Zeitler JA, Taday PF, Newnham DA, Pepper M, Gordon KC, Rades T. 2006. Terahertz pulsed spectroscopy and imaging in the pharmaceutical setting. J Pharm Pharmacol (in press).

15. Fitzgerald AJ, Cole BE, Taday PF. 2005. Nondestructive analysis of tablet coating thicknesses using terahertz pulsed imaging. J Pharm Sci 94:177-183.

16. Conte U, Maggi L, Colombo P, Lamanna A. 1993. Multilayered hydrophilic matrices as constant release devices (Geomatrix(Tm) Systems). J Control Release 26:39-47.

17. Piao ZS, Tani M, Sakai K. 2000. Carrier dynamics and terahertz radiation in photoconductive antennas. Jpn J Appl Phys Part 1-Regular Pap Short Notes Rev Pap 39:96-100.

18. Kono S, Tani M, Gu P, Sakai K. 2000. Detection of up to $20 \mathrm{THz}$ with a low-temperature-grown GaAs photoconductive antenna gated with $15 \mathrm{fs}$ light pulses. Appl Phys Lett 77:4104-4106.

19. Baker C, Gregory IS, Tribe WR, Bradley IV, Evans MJ, Withers M, Taday PF, Wallace VP, Linfield EH, Davies AG, Missous M. 2003. Terahertz pulsed imaging with $1.06 \mathrm{mu}$ m laser excitation. Appl Phys Lett 83:4113-4115.

20. Kirsch JD, Drennen JK. 1995. Determination of film-coated tablet parameters by near-infrared spectroscopy. J Pharm Biomed Anal 13:12731281.

21. Kirsch JD, Drennen JK. 1996. Near-infrared spectroscopic monitoring of the film coating process. Pharm Res 13:234-237. 